\title{
Os benefícios do magnésio em praticantes de exercício físico: um estudo de revisão bibliográfica integrativa
}

\author{
The benefits of magnesium in exercise practitioners: integrative literature review study \\ Los beneficios del magnesio en los practicantes del ejercicio: un estudio de revisión de la literatura
}

integradora

Recebido: 09/08/2021 | Revisado: 17/08/2021 | Aceito: 18/08/2021 | Publicado: 22/08/2021

Jefferson Cardoso da Silva

ORCID: https://orcid.org/0000-0002-6872-4940

Universidade Potiguar, Brasil

E-mail: jefferson_cardos@hotmail.com

Glayciana Mattos dos Santos

ORCID: https://orcid.org/0000-0002-7269-9872 Universidade Potiguar, Brasil

E-mail: glayciana.mattos@hotmail.com

Marcília Ingrid Lima Barroso Nunes

ORCID: https://orcid.org/0000-0002-5871-3930 Universidade Potiguar, Brasil

E-mail: prof.marciliabarroso@gmail.com

Paloma Katlheen Moura Melo

ORCID: https://orcid.org/0000-0002-9265-038X Faculdade do Vale do Jaguaribe, Brasil

E-mail: palomakatlheen@hotmail.com

Roque Ribeiro da Silva Júnior

ORCID: https://orcid.org/0000-0001-9517-6823 Universidade do Estado do Rio Grande do Norte, Brasil

E-mail: roquejunior@alu.uern.br

Francisco Medeiros de Azevedo Filho

ORCID: https://orcid.org/0000-0003-2725-1945 Universidade Potiguar, Brasil

E-mail: medeirosfilhoef@ hotmail.com

Andreza Dayanne França Freire Medeiros

ORCID: https://orcid.org/0000-0001-6978-4542 Universidade Potiguar, Brasil

E-mail: andrezadayane@hotmail.com

Francisco Sérgio Lopes Vasconcelos Filho

ORCID: https://orcid.org/0000-0003-3140-0085

Universidade Federal do Cariri, Brasil

E-mail: sergio.vasconcelos@ufca.edu.br

\begin{abstract}
Resumo
Introdução: $\mathrm{O}$ magnésio participa do metabolismo energético, da regulação dos transportadores de íons, na defesa antioxidante e aspectos metabólicos importantes para o desempenho na prática esportiva. Objetivo: O presente estudo objetiva descrever os benefícios do magnésio e suas principais fontes alimentares, apontar os danos à saúde decorrentes da deficiência do mineral e correlatar a atuação do magnésio em praticantes de exercício físico. Métodos: Trata-se de um estudo de revisão integrativa da literatura, contribuindo para o processo de sistematização e análise dos resultados a partir de outros estudos independentes sobre o tema tratado. Resultados: O magnésio é um antioxidante envolvido em mais de 300 reações metabólicas e bioenergéticas no corpo, o exercício físico tem como incumbência levar à redistribuição do magnésio no organismo durante e após a prática, variando de acordo com o tipo de exercício, e o estado nutricional do indivíduo, embora seja um mineral contido em alimentos de fácil acesso, existem inadequação na sua ingestão com o seu consumo reduzido pela população. Considerações finais: Torna-se evidente a importância da ingestão do magnésio uma vez que o mineral está diretamente ligado a vários processos que afetam a função muscular, no entanto há grande carência de estudos sobre a temática.
\end{abstract}

Palavras-chave: Magnésio; Exercício físico; Metabolismo; Nutrientes.

\section{Abstract}

Introduction: Magnesium participates in energy metabolism, regulation of ion transporters, antioxidant defense and important metabolic aspects for performance in sports practice. Objective: This study aims to describe the benefits of 
magnesium and its main food sources, point out the damage to health resulting from the mineral deficiency and correlate the role of magnesium in people who exercise. Methods: This is an integrative literature review study, contributing to the process of systematization and analysis of results based on other independent studies on the topic discussed. Results: Magnesium is an antioxidant involved in more than 300 metabolic and bioenergetic reactions in the body, physical exercise is intended to lead to the redistribution of magnesium in the body during and after practice, varying according to the type of exercise, and the state of the individual, although it is a mineral contained in easily accessible foods, there is an inadequacy in its intake with its reduced consumption by the population. Final considerations: It is evident the importance of magnesium intake, since the mineral is directly linked to several processes that affect muscle function, however there is a great lack of studies on the subject.

Keywords: Magnesium; Exercise; Metabolism; Nutrients.

\section{Resumen}

Introducción: El magnesio participa en el metabolismo energético, la regulación de los transportadores de iones, la defensa antioxidante y aspectos metabólicos importantes para el rendimiento en la práctica deportiva. Objetivo: Este estudio tiene como objetivo describir los beneficios del magnesio y sus principales fuentes alimenticias, señalar el daño a la salud derivado de la deficiencia de minerales y correlacionar el papel del magnesio en las personas que hacen ejercicio. Métodos: Se trata de un estudio de revisión integradora de la literatura, que contribuye al proceso de sistematización y análisis de resultados a partir de otros estudios independientes sobre el tema tratado. Resultados: El magnesio es un antioxidante involucrado en más de 300 reacciones metabólicas y bioenergéticas en el cuerpo, el ejercicio físico está destinado a conducir a la redistribución del magnesio en el cuerpo durante y después de la práctica, variando según el tipo de ejercicio y el estado de el individuo, si bien es un mineral contenido en alimentos de fácil acceso, existe una inadecuación en su ingesta con su reducido consumo por parte de la población. Consideraciones finales: Es evidente la importancia de la ingesta de magnesio, ya que el mineral está directamente ligado a varios procesos que afectan la función muscular, sin embargo hay una gran falta de estudios sobre el tema.

Palabras clave: Magnesio; Ejercicio físico; Metabolismo; Nutrientes.

\section{Introdução}

Na pesquisa Silva (2013) nos últimos anos tem-se observado cada vez mais o papel dos micronutrientes no desempenho físico, pois tem sido alvo de pesquisas correlacionadas ao exercício físico. O mineral magnésio, em particular, participa do metabolismo energético, da regulação dos transportadores de íons, na defesa antioxidante e aspectos metabólicos importantes para o desempenho na prática esportiva.

Nunes (2011) explica que exercício físico tem a capacidade de aumentar a utilização de antioxidantes, e dentre essas substâncias antioxidantes temos o magnésio que participa do metabolismo energético, da regulação dos transportadores de íons, da contração muscular e da coagulação sanguínea, também possui importante função na defesa contra os radicais livres.

Quando levamos em consideração que a deficiência dietética de determinados nutrientes pode afetar diretamente tal fenômeno biológico, podemos destacar o magnésio, no qual até o momento, pouco tem sido discutido a respeito do seu efeito sobre o metabolismo oxidativo durante o exercício físico (Amorin \& Tirapegui, 2008).

Diversos estudos têm sido conduzidos na perspectiva de elucidar o papel de alguns nutrientes envolvidos no metabolismo energético ou com função antioxidante no exercício físico. Assim, é importante destacar a participação do magnésio como um nutriente capaz de interferir diretamente dentre estas duas atividades (Melo, 2020).

Diante do exposto, o presente estudo tem como objetivo descrever os benefícios do magnésio e suas principais fontes alimentares, apontar os danos à saúde decorrentes da deficiência do mineral e correlatar a atuação do magnésio em praticantes de exercício físico.

\section{Metodologia}

Trata-se de um estudo de revisão integrativa da literatura, uma vez que ela contribui para o processo de sistematização e análise dos resultados, visando a compreensão de determinado tema, a partir de outros estudos independentes.

Foram adotados os seguintes critérios de inclusão: estudos com textos completos disponíveis para análise e que estejam indexados eletronicamente nos sites PubMed e Scielo; artigos na língua portuguesa, espanhola e/ou inglesa, como 
também em livro. Assuntos que abordassem uma das variáveis estudadas, tais como benefícios do magnésio, fontes alimentares de magnésio, carência de magnésio, formas de suplementar o magnésio e atuação do magnésio na prática de exercício físico. Os termos de pesquisa utilizados foram: magnésio, exercício físico, metabolismo, nutrientes.

Os critérios de exclusão adotados neste estudo foram: artigos não disponíveis gratuitamente; artigos disponíveis apenas em resumo; publicações como cartas, comentários e editoriais.

Do material obtido, 33 artigos, procedeu-se à leitura minuciosa de cada resumo/artigo, destacando aqueles que responderam ao objetivo proposto por este estudo, a fim de organizar e tabular os dados. Seguindo os critérios de inclusão, 26 estudos foram selecionados para análise, os quais são referenciados no presente texto.

As etapas de busca e seleção dos artigos para leitura do texto completo e elegibilidade para análise qualitativa foram realizados pelos autores no período de março a abril de 2021, solucionando os conflitos em conjunto.

\section{Resultados e Discussão}

Inicialmente, obteve-se um quantitativo de 197 publicações provenientes de periódicos científicos. Após exclusão de duplicatas e avaliação de título e temática, o número de artigos diminuiu para 101. A partir disso, foi feita leitura dos resumos e/ou textos completos, e por não serem compatíveis com os critérios de inclusão/exclusão eliminou-se mais 68 artigos. Portanto, confirmando-se a elegibilidade pela leitura detalhada pelo manuscrito e considerando a aproximação com a questão norteadora deste estudo, estabeleceu-se um quantitativo de 33 artigos (figura 1).

Figura 1 - Fluxograma de seleção dos estudos.

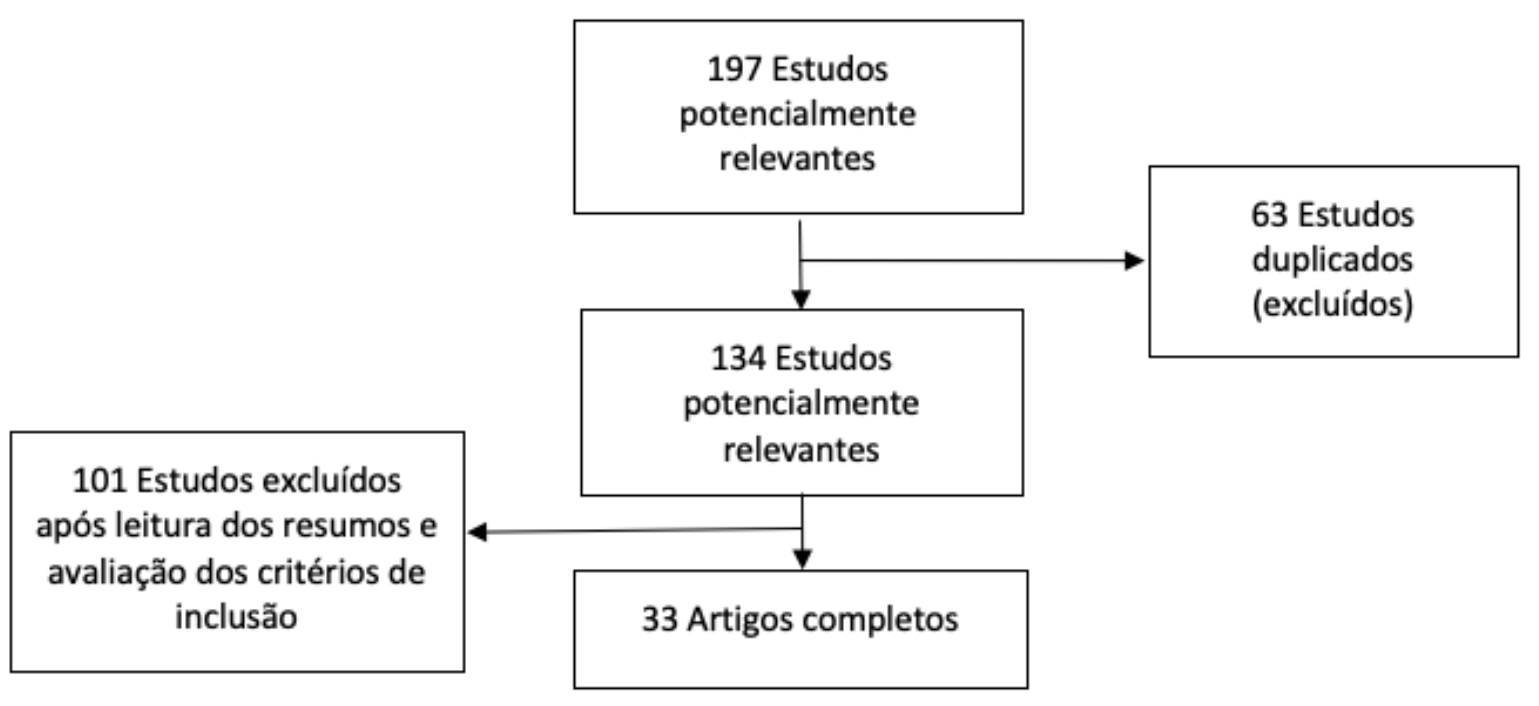

Fonte: Autores (2021).

\subsection{Atuação do magnésio}

Segundo Silva (2013), o magnésio atua como um cofator de centenas de reações enzimáticas, especificamente para as enzimas que utilizam nucleotídeos como cofator ou substrato. É requisitado para síntese de proteínas e ácidos nucleicos e para a ligação de substâncias na membrana plasmática. Frequentemente modula o transporte iônico por bombas, carreadoras e canais, modulando a transdução de sinais e as concentrações citossólicas de cálcio e potássio.

Seu papel na regulação da via glicolítica e no ciclo do ácido cítrico (ciclo de Krebs) é de suma importância, pois é necessário tanto na geração de energia aeróbica, quanto anaeróbica. Na glicose atua indiretamente como complexo Mg-ATP ou diretamente como um cofator enzimático (Amorim, 2002). 
Estudos recentes têm sido conduzidos na perspectiva de elucidar o papel de alguns nutrientes envolvidos no metabolismo energético, na função antioxidante, da regulação dos transportes de íons e da contração muscular (Mendiola \& Cruz, 2014; Gomes et al., 2016).

Rezende et al. (2019) ressaltam que é importante destacar a participação do magnésio como um nutriente que apresenta essas funções, pois é um antioxidante envolvido em mais de 300 reações metabólicas e bioenergéticas no corpo, ajudando na manutenção da função nervosa e muscular, no ritmo cardíaco, pressão sanguínea, relaxamento e contração muscular, transporte transmembranquial, no sistema imune, nas concentrações de glicose no sangue, na homeostase insulínica e glicêmica e na síntese de adenosina trifosfato, proteínas e ácidos nucleicos.

O magnésio atua ainda na integridade óssea, na estabilidade da membrana neuromuscular e cardiovascular, na manutenção do tônus vasomotor e como regulador fisiológico da função hormonal e imunológica (Melo, 2020).

\subsection{Exercício físico e magnésio}

O exercício físico tem como incumbência levar à redistribuição do magnésio no organismo, variando de acordo com o tipo de exercício, (no qual são a intensidade, seleção e ordem dos exercícios, intervalo de recuperação, velocidade do movimento e frequência) e o seu estado nutricional, no qual influenciam no resultado da natureza desta redistribuição. Além disso, os exercícios de alta intensidade e de curta duração aumentam a concentração plasmática de magnésio em 5\% a 15\%, retornando aos seus valores iniciais 24 horas após os exercícios. Já nos exercícios de baixa intensidade não há alterações da concentração plasmática de níveis de magnésio (Amorin \& Tirapegui, 2008).

Estudos afirmam que a perda de massa muscular seria correspondente ao aumento do magnésio sérico, no qual se refere à ingestão, absorção e excreção de níveis de magnésio, logo após o exercício, no entanto, no exercício prolongado ocorre redução da concentração sérica. Estes parâmetros geralmente retornam aos valores iniciais, provavelmente devido ao movimento do magnésio em direção a outros compartimentos e devido ao aumento da excreção pela urina e suor, dessa forma, o magnésio é redistribuído no exercício para os locais com maior necessidade metabólica para a produção de energia ou na prevenção do estresse oxidativo (Nielsen \& Lukaski, 2006; Amorim, 2007; Amorin \& Tirapegui, 2008).

Rezende et al. (2019) abordam o fluxo de magnésio, no qual ocorre durante e após o exercício físico, o magnésio é transferido do soro em direção aos adipócitos e à musculatura esquelética ativa durante o exercício físico, o grau de passagem do magnésio extracelular para estes órgãos é modulado pelo nível de produção de energia aeróbia, logo após o exercício aeróbio, ocorre a redistribuição do magnésio dos tecidos para a circulação. Assim, o magnésio é então mobilizado para o osso, para os tecidos moles, para o músculo e para o adipócito, com a finalidade de restaurar as concentrações de magnésio plasmático prévias ao exercício.

Com o exercício físico é possível reduzir a concentração plasmática do magnésio no sangue e na urina, como também, transportá-lo para atuar nas demandas do músculo esquelético durante o exercício físico (Kezianne, 2019).

Levando em consideração as funções críticas no metabolismo, a regulação, em casos de deficiência se dá através da retenção do mineral, sugerindo que, para um status sérico normal, não haveria alteração da homeostase do magnésio, sendo que nesses casos o mineral não seria capaz afetar a performance (Nielsen \& Lukaski, 2006).

\subsection{Principais fontes de magnésio e sua suplementação}

O magnésio é um mineral encontrado em diversos alimentos, porém em concentrações variadas, pois é um componente da estrutura da clorofila, no qual as principais fontes são vegetais verdes escuros, cereais integrais, frutas secas, oleaginosas e tubérculos como a batata (Amorim, 2002).

Dentre os alimentos que são fonte de magnésio estão: as frutas como uva, banana e abacate; grãos e derivados como a 
granola, gérmen de trigo e aveia; sementes e nozes como gergelim, amendoim, girassol e castanhas, além de leite, soja, grão de bico, pão, peixes, batata, beterraba, couve e espinafre (Ramires, 2014).

Embora seja um mineral contido em alimentos de fácil acesso, é possível afirmar a inadequação na ingestão de diversos nutrientes entre os indivíduos, estando entre eles o magnésio, como sendo um dos minerais com consumo reduzido pela população. Existem ainda fatores inibidores do processo de absorção do magnésio, como a presença na dieta de alimentos ricos em fitatos, oxalatos, fosfatos e fibras alimentares, e promotores, tais como a lactose e carboidratos (Morais, 2017; Severo et al., 2015).

Morais (2017) complementa que a deficiência de magnésio pode decorrer da ingestão inadequada ou ainda da excreção aumentada, sendo a homeostase desse nutriente em nosso organismo regulada principalmente pelos rins, no qual desencadeia sérias consequências ao nosso organismo, dentre elas ao aumento da peroxidação lipídica, tendo em vista a diminuição da atividade antioxidante.

Considerando a importância do magnésio no metabolismo e manutenção da homeostase do organismo, referente a escassez de dados sobre o consumo de alimentos ricos do mineral em questão (Severo et al., 2015).

Temos determinações em relação as recomendações nutricionais do mineral em questão, que têm como objetivo atender às necessidades humanas, incluindo os indivíduos idosos (Marques, 2004).

Vitolo (2008) afirma que a suplementação de nutrientes é necessária quando não há oportunidades de incluir nas refeições a quantidade necessária que o indivíduo necessita diariamente.

As recomendações nutricionais estabelecidas e utilizadas como referência para orientação dietética são as DRI (Ingestão Alimentar de Referência ou Dietary Reference Intakes) que para o magnésio é de 400 a 420 e 310 a 320mg diários para homens e mulheres adultas, respectivamente. Enquanto os valores de referência da RDC $n^{\circ} 269$ da ANVISA a recomendação de ingestão diária de magnésio e de 260 mg para mulheres e homens (Brasil, 2005).

Segundo Amorin e Tirapegui, (2008) levantaram o questionamento a respeito das atuais Ingestões Dietéticas de Referência (Dietary Reference Intakes - DRI) em nosso país para magnésio estar no fato de os valores citados acima terem sido estabelecidos praticamente a partir de um único estudo.

\subsection{Sintomas da carência de magnésio no sangue}

Os micronutrimentos são essenciais para a saúde do indivíduo e, embora existindo nas mais diversas fontes alimentares, deve-se ter atenção as patologias associadas ao seu défice e forma de as prevenir ou colmatar (Marques, 2004).

A deficiência de magnésio pode aumentar a produção de radicais livres, levando a alterações nas membranas celulares e ao aumento na concentração de cálcio intracelular. Este aumento dificulta a contração muscular e ativa enzimas importantes na produção de eicosanoides, a ação conjunta desses mecanismos facilita a suscetibilidade a lesões e, consequentemente, prejudica o desempenho físico (Amorin \& Tirapegui, 2008).

Além disso, com a redução dos índices de magnésio, o cálcio aumenta no espaço intracelular, podendo resultar em cãibras, hipertensão e vasoespasmos coronarianos e cerebrais. Além disso, os baixos níveis de magnésio têm sido associados a doenças crônicas, como Alzheimer, resistência à insulina e diabetes mellitus tipo 2, enxaqueca e déficit de atenção e hiperatividade e doenças cardiovasculares de acordo com os estudos de Rezende et al. (2019).

A deficiência em magnésio pode prejudicar o fornecimento de oxigénio e, assim, reduzir a capacidade de realizar e concluir exercícios submáximos, o que, por sua vez, irá reduzir a performance (Amorim \& Loureiro, 2014).

O magnésio é fundamental para a formação e manutenção da estrutura óssea dos tecidos, sendo essencial na prevenção de patologias como a osteoporose, doença progressiva que resulta na perda de massa óssea ao longo da vida (Santana, 2015). Segundo a OMS a doença atinge a cada uma em três mulheres e um a cada cinco homens acima dos 50 anos, 
no mundo, na maioria dos casos a osteoporose se desenvolve de forma imperceptível, sem sintomas ou dor, sendo detectada somente quando ocorre a fratura de um osso (Gali, 2001).

A diminuição dos níveis de magnésio clinicamente significativa, no qual é classificado pela concentração plasmática de magnésio $<1,8 \mathrm{mg} / \mathrm{dL}(<0,70 \mathrm{mmol} / \mathrm{L}$ ) pode resultar na hipomagnesemia (comum em pacientes hospitalizados), afetando principalmente órgãos do corpo, especialmente o coração, músculos e rins (Barbosa, 2015; Berríos, 2006).

As causas da hipomagnesemia incluem ingestão inadequada de magnésio por pacientes com má nutrição e é agravada pelo aumento da excreção urinária exacerbada decorrentes do uso de diuréticos, consequentemente aumentando a excreção urinária de magnésio, uso de fármacos (como furosemida) durante o tratamento do paciente, nestes casos a hipomagnesemia geralmente desaparece com a interrupção da terapia (Barbosa, 2015; Berríos, 2006).

\section{Considerações Finais}

Torna-se evidente a importância da ingestão do magnésio por meio de suas diversas fontes, uma vez que o mineral está diretamente ligado a vários processos que afetam a função muscular, grandes perdas urinárias e de suor, assim como tratamentos medicamentosos podem aumentar a necessidade de magnésio e ainda trazer sérias consequências à saúde do indivíduo decorrentes de sua deficiência no organismo.

O exercício físico é capaz de induzir a redistribuição de magnésio no corpo durante e após exercício físico para acomodar as necessidades metabólicas do organismo, havendo variações de acordo com o nível de exercício físico que esteja sendo praticada e seu estado nutricional.

Sugere-se para trabalhos futuros a comparação por meio de exames laboratoriais entre grupos de indivíduos que apresentem deficiência de magnésio que estejam suplementando e praticando exercício físico em relação a indivíduos que façam a suplementação, mas que não estejam inseridos em um programa regular de exercício físico.

\section{Referências}

Amorim, A. G. (2007). Efeito da deficiência dietética de magnésio no metabolismo oxidativo de tecidos de ratos submetidos a protocolo de treinamento periodizado. Universidade de São Paulo. Faculdade de ciências farmacêuticas.

Amorim, A. G. (2002). Magnésio na dieta de praticantes de musculação. Universidade de São Paulo, Faculdade de ciências farmacêuticas. https://www.teses.usp.br/teses/disponiveis/9/9132/tde29012015154119/publico/AlineGAmorim_M.pdf

Amorim, S., \& Loureiro, N. (2014). Magnésio e Exercício físico. Federação portuguesa de ciclismo. https://www.fpciclismo.pt/fiche irossite/16102014094602.pdf

Amorin, A. G., \& Tirapegui, J. (2008). Aspectos atuais da relação entre exercício físico, estresse oxidativo e magnésio. Rev. Nutr., Campinas, 21 (5),563-575.

Barbosa, E. B. (2015). Reposição de magnésio endovenoso em pacientes com hipomagnesemia assintomática não severa para prevenção de lesão renal aguda. Universidade do Extremo Sul Catarinense. Dissertação apresentada ao programa de Pós-Graduação em Ciências da Saúde. Criciúma/SC.

Berríos, H. R. (2006). Hipomagnesemia. Nephrology Fellow. Renal-Electrolyte Division. Department of Medicine. University of Pittsburgh Medical Center. Pittsburgh, Pennsylvania, USA. Anales de la Facultad de Medicina Universidad Nacional Mayor de San Marcos. 4(1), 11-24.

BRASIL. (2005). Resolução RDC n ${ }^{\circ}$ 269, de 22 de setembro de 2005. Aprova o regulamento técnico sobre a ingestão diária recomendada (idr) de proteína, vitaminas e minerais constante do anexo desta Resolução. Órgão emissor: ANVISA - Agência Nacional de Vigilância Sanitária. 3(2), 1-54.

Gali, J. C. (2001). Osteoporose. Acta ortop bras 9(2), 1-12. http://www.scielo.br/pdf/aob/v9n2/en_v9n2a07.pdf

Gomes, A. L. (2009). O benefício da prestação continuada: uma trajetória de retrocessos e limites - construindo possibilidades de avanços? In: Seminário internacional: mínimos de cidadania e benefícios a idosos e pessoas deficientes - Brasil, França e Portugal. São Paulo: FAPESP.

Gomes S. F., Silva, F. C., Pinheiro V., \& Ana C. (2016). Efeito do consumo de frutas ricas em flavonoides sobre mediadores inflamatórios, bioquímicos e antropométricos relacionados ao metabolismo energético. Nutr. clín. diet. hosp. 36(3), 170-180.

Kezianne R. C., Matheus P. D., Romulo V. T, Gustavo C. S., Paulo A. N., Marcela G. U., \& Breno G. A. T. C. (2019). Relação de magnésio sérico e capacidade de sprints repetidos em crianças. Motricidade. Edições Desafio Singular. 15(3) 23-27. https://www.researchgate.net/profile/Romulo_T eixeira/publication/340 874800_Relacao_de_magnesio_serico_e_capacidade_de_sprints_repetidos_em_criancas/links/5ea1ddd9a6fdcc88fc39fe2f/Relacao-demagnesio-serico-e-capacidade-de-sprints-repetidos-em-criancas.pdf 
Marques, A. T. M. P. (2004). Nutrição no idoso a problemática dos micronutrimentos. Faculdade de Ciências da Nutrição e Alimentação Universidade do Porto. Monografia: Nutrição Clínica em idosos.

Matsudo S. M., Matsudo V. K., \& Neto T. L. B. (2001). Atividade física e envelhecimento: aspectos epidemiológicos. Revista Bras Med Esportiva. 7(1), 1-14.

Melo, S. R. S., Santos L. R., Silva, T. M., Cardoso, B. E. P., Araújo, D. S., Sousa, T. G. V., Sousa, M. P., Severo, J. S., \& Marreiro D. N. (2020). Suplementação com magnésio sobre a performance de atletas: uma revisão sistemática. Research, Society and Development, 9(1), 1179-11754.

Mendiola A. V., \& Cruz I. S. (2014). Metabolismo energético y cáncer. VERTIENTES Revista Especializada en Ciencias de la Salud, 17(2),108-113.

Monteiro, R. E. G., \& Coutinho, D. J. G. (2020). Uma breve revisão de literatura sobre os idosos, o envelhecimento e saúde. Braz. J. of Develop. 6(1), 23582368 .

Morais, L. L. (2017). Micronutrientes antioxidantes no exercício físico: uma revisão da literatura. Vitória de Santo Antão. 30 folhas. UFPE. Centro Acadêmico de Vitória Curso de Graduação em Nutrição.

Nielsen F. H., \& Lukaski H. C. (2006). Update on the relationship between magnesium and exercise. Magnes Res. 19(3), 180-190.

Nunes, L. A. S., Silva, F. O. C., Ceglio, A. C., \& Venturini, T. S. (2011). Efeitos da Suplementação com Panax ginseng Sobre Parâmetros de Estresse Oxidativo e Lesão Muscular Induzidos por Exercício Exaustivo Agudo em Ratos. Revista ciência em saúde. 1(1), 15-26.

Ramires, A. D. (2014). Influência do calcio e magnésio sobre o ganho de peso corporal: uma abordagem experimental em ratos wistar em crecimento. UFMS. https://repositorio.ufms.br:8443/jspui/handle/123456789/2082

Rezende, M. P., Ramos, R. C. S., Paula, C. R., Pelazza, B. B., \& Silva, M. I. A. (2019). Influênciaa dos níveis de magnésio na fadiga muscular: uma revisão sistemática. Saúde coletiva. 09(49), 1-16.

Santana, J. S. (2015). Eficácia do carbonato de cálcio em pacientes idosos com osteoporose: uma visão teórica. Faculdade Maria Milza Curso de Bacharelado em Farmácia. http://131.0.244.66:8082/jspui/bitstream/123456789/1147/1/Monografia\%20joelson\%20pdf.pdf

Santos, F. H., Andrade, V. M., \& Bueno, O. F. A. (2014). Envelhecimento: um processo multifatorial. Psicologia em estudo. Maringá, 14(1), 3-10.

Santos, D. A., Matias, C. N., Monteiro, C. P., Silva, A. M., Rocha, P. M., Minderico, C. S., \& Laires, M. J. (2019). Magnesium intake is associated with strength performance in elite basketball, handball and volleyball players. Magnesium Research, 24(4), 215-219.

Severo, J. S., Morais, J. B. S., Freitas, T. E. C., Cruz, K. J. C., Oliveira, A. R. S., Poltronieri, F., \& Marreiro, D. N. (2015). Aspectos Metabólicos e Nutricionais do Magnésio. Nutr. clín. diet. Hosp. 35(2),67-74.

Silva, R., Beserra, B. T. S., Oliveira, A. R., Barbosa, A. M., Silva, J. C., Poltronieri, F., \& Nascimento, D. M. (2013). Relação entre exercício físico, estresse oxidativo e magnésio. Nutrição em Pauta.

Vitolo, M. R. (2008). Nutrição da gestação ao envelhecimento. Ed Rubia. 